Address for Correspondence: Dr. Yin-Fei Zhang, Central Hospital of Jiading District, Shanghai 200025, China. E-mail: zyyf@msn.com

\begin{tabular}{|l|}
\hline Access this article online \\
\hline $\begin{array}{l}\text { Website: } \\
\text { www.intern-med.com }\end{array}$ \\
\hline DOI: \\
10.1515/jim-2013-0010 \\
\hline Quick Response Code: \\
\hline \\
\\
\hline
\end{tabular}

\title{
Association between blood lipid levels and carotid intima-media thickness in Shanghai urban population
}

\author{
Ya-Ya Guan' ${ }^{1}$, Mian $\mathrm{Li}^{2}$, Xiao-Ou Chen ${ }^{1}$, Wei Yang${ }^{1}$, Ming-Ming Tang ${ }^{1}$, Yu-Hong \\ Chen $^{2}$, Yin-Fei Zhang ${ }^{*}$ \\ ${ }^{1}$ Central Hospital of Jiading District, Shanghai 201800, China; ${ }^{2}$ Shanghai Clinical Center for Endocrine and \\ Metabolic Diseases, Department of Endocrinology and Metabolism of Ruijin Hospital, School of Medi- \\ cine, Shanghai Jiao Tong University Shanghai Institute of Endocrine and Metabolic Diseases, Shanghai \\ 200025, China
}

\section{ABSTRACT}

Objective: The objective of the following study is to investigate the relationship between blood lipid levels and carotid intima-media thickness (CIMT) in Shanghai urban population. Materials and Methods: A total of 2504 residents over 40 years old in Jiading District of Shanghai municipality were divided into two groups in terms of the CIMT value $0.7 \mathrm{~mm}$ : Thickened group $(n=478)$ and normal group $(n=2026)$. All the patients received examinations of height, weight, waist, blood pressure, blood lipid level, hepatic and renal function, oral glucose tolerance test and carotid artery B-ultrasonography. Results: In comparison with those in CIMT normal group, the proportion of males, ages, body mass index, systolic blood pressure and levels of fast blood glucose, total cholesterol (TC), triglyceride (TG) and lowdensity lipoprotein cholesterol (LDL-C) were increased, but the levels of fast insulin were decreased (all $P<0.05$ ). The risks of CIMT thickness were elevated by $19 \%$ and $25 \%$ as every increase in the standard deviation of the TC and LDL-C levels respectively (95\% of confidence interval [Cl] 1.08-1.31; 95\% Cl 1.13-1.37). Statistical analysis showed a significant correlation between CIMT thickness and TC/LDL-C. The levels of TG and high-density lipoprotein cholesterol were not associated with CIMT thickness. Conclusions: Levels of blood TC and LDL-C have significant correlations with the CIMT thickness in Shanghai urban population.

Key words: atherosclerosis, carotid intima-media thickness, lipid

\section{INTRODUCTION}

Atherosclerosis is an important risk factor for cardiovascular and cerebrovascular diseases. ${ }^{[1]}$ Lipid metabolic disorders play an important role in the occurrence and progression of atherosclerosis. ${ }^{[2]}$ Ultrasound measurement of carotid intimamedia thickness (CIMT), a noninvasive method to evaluate progression of atherosclerosis at early stages, is considered as an early marker for systemic atherosclerosis. ${ }^{[3]}$ This study aims to investigate the correlation between lipid levels and CIMT in Shanghai urban community population aged over 40 years old.

\section{MATERIALS AND METHODS}

\section{Subjects}

Cluster sampling was applied to the investigation of diabetes and related diseases among residents in Juyuan New Area of Shanghai Jiading District from March to July, 2010. A total of 2,504 subjects aged over 40 years old included in this study had finished a questionnaire survey, physical examinations, B-mode ultrasound of the carotid artery and biochemical detections including fasting blood glucose, fasting insulin, blood lipid, liver function and renal function tests. This study was approved by Ethic Committee of Ruijin Hospital, School 
of Medicine of Shanghai Jiao Tong University and written informed consent was obtained from all participants.

\section{Methods}

\section{History taking}

Standardized questionnaire survey was performed to collect information of all participants, including past medical history, medication history, family history of diabetes, smoking and alcohol consumption, physical exercise, work and financial situation and complications. All the investigators received uniform training.

\section{Physical examination}

All subjects received routine medical examinations including height, weight, waist circumference and resting blood pressure. Height was measured to the nearest 0.1 $\mathrm{cm}$; while weight was measured with accuracy of $0.1 \mathrm{~kg}$. Body mass index (BMI) was calculated as the weight in kilograms divided by the square of the height in meters. Waist circumference was measured at the umbilicus. Blood pressure was measured in the supine position on the nondominant arm after at least 15 min of rest using Omron digital blood pressure monitor (OMRON Model HEM-752 FUZZY, Omron Corporation, Dalian) and the average of three readings was used for data analysis.

\section{Biochemical detection}

Fasting oral glucose tolerance test using $75 \mathrm{~g}$ anhydrous glucose was performed in the morning, 2-h blood samples were collected from participants. Automatic biochemical analyzer was used for enzymatic tests of blood lipid [triglyceride (TG), total cholesterol (TC), low-density lipoprotein cholesterol (LDL-C), high-density lipoprotein cholesterol (HDL-C)]. Glucose oxidase method was applied to the measurement of fasting blood glucose (ADVIA-1650 biochemical analyzer, Bayer Company, Germany). Electrochemiluminescence method was used for the measurement of fasting insulin (Roche Company, Switzerland).

\section{B-mode ultrasound of the carotid artery}

MYLAD30 color Doppler ultrasonography from Esoate Company was used with $5-10 \mathrm{MHz}$ probe. Minimal gain was adjusted to visualize the lumen-intimal and medialadventitial interfaces defining intima-media thickness (IMT) in the far wall. Subjects lay in horizontal and overhead position, with head extended and turned to the contra-lateral side and a full exposure of the carotid artery. Cross-sectional scanning of right arteria carotis communis was performed first, from the origin of arteria carotis communis to where the image of the internal carotid artery disappeared, to get a general understanding of the arrangement of arteria carotis communis, intimamedia, formation and location of possible atherosclerotic plaque. Then a longitudinal scan of right arteria carotis communis was performed to measure IMT of arteria carotis communis located at the proximal end $1.0 \mathrm{~cm}$ from the bifurcation of arteria carotis communis. Plaques should be avoided during measurement. Intima-media thickness of the left arteria carotis communis was measured in the same way, followed by analysis using the maximum of values from the left and right side. Carotid artery ultrasound was performed by professional physicians. The CIMT values $>0.7 \mathrm{~mm}$ above $1 / 5$ sites was defined as CIMT thickening.

\section{Statistical methods}

The SAS 8.1 software package was used for analysis. Normally distributed measurement data were represented by mean \pm standard deviation and non-normal measurement data were represented by median M $(95 \%$ confidence interval [CI]), all of which were statistically analyzed after a natural logarithm conversion. For continuous variables, ANOCA analysis was applied to intergroup comparison and Chi-square test was used for constituent ratio analysis. Linear regression model was built to determine the CIMT-related factors. Logistic regression analysis with two categories was employed for risk assessment. $P$ is considered to be statistically significant if $<0.05$.

\section{RESULTS}

\section{General information of subjects}

A total of 2,504 subjects were divided into normal CIMT group (2,026 cases) and thickened CIMT group (478 cases). Compared with normal CIMT group, thickened CIMT group had a higher proportion of male subjects, older age, larger BMI and waist circumference, higher systolic blood pressure (SBP), higher levels of fasting blood glucose, TC, TG and LDL-C and lower levels of fasting insulin $(P<0.05$ for all). But no statistically significant difference in diastolic blood pressure (DBP) or HDL-C was found between the two groups.

\section{Analysis of related factors}

Linear regression analysis showed that CIMT was significantly correlated to the female gender, SBP, DBP, fasting blood glucose, fasting insulin, TC, TG and LDL-C $(P<0.05$ for all $)$. Correlation of CIMT with waist circumference, BMI and HDL-C was not significant.

\section{Correlation analysis between CIMT thickening and indicators of blood lipids}

The risk of CIMT thickening along with every standard deviation increase of blood lipid indicators was assessed in a multi-factor logistic regression model, using the absence or presence of CIMT thickening as the dependent variable and TC, TG, LDL-C, HDL as the respective independent variable. For every standard deviation increase of TC, the 
risk of CIMT thickening increased and OR $(95 \% \mathrm{CI})$ was 1.19 (1.08-1.31) without adjustment for other variables. After adjustment for confounding factors including age, gender, BMI, waist circumference, SBP, DBP, fasting blood glucose and fasting insulin, the correlation between TC and CIMT thickening was still found, with OR $(95 \% \mathrm{CI})$ of 1.32 (1.16-1.49). For every standard deviation increase of LDL-C, the risk of CIMT thickening increased and OR (95\% CI) was 1.25 (1.13-1.37) without adjustment for other variables. After adjustment for confounding factors including age, gender, BMI, waist circumference, SBP, DBP, fasting blood glucose and fasting insulin, the correlation between LDL-C and CIMT thickening could still be found, with OR $(95 \% \mathrm{CI})$ of 1.42 (1.26-1.61). No significance was found in the correlation of the increased TG or HDL-C with CIMT thickening [Table 1].

\section{DISCUSSION}

Atherosclerosis is a kind of systemic diffuse lesions, which can lead to structural and functional alterations of various vital organs in the human body, especially the heart, brain and kidney. ${ }^{[4]}$ Ultrasound measurement of CIMT can provide evidence for the diagnosis of subclinical atherosclerosis and facilitate the assessment as well as early intervention of subclinical atherosclerosis. ${ }^{[5]}$ Currently, atherosclerosis is believed to be a multi-factorial disease and lipid metabolic disorders are the key pathogenic factor, playing an important role in its development. ${ }^{[6]}$

Our study found that the increased TC, TG and LDL-C in thickened CIMT group were statistically significant in comparison with normal CIMT group. In the multi-factor logistic regression model, the risk of CIMT thickening rose along with the increased TC and LDL-C, which was still correlated independently with CIMT thickening after adjustment for confounding factors including age, gender, BMI, waist circumference, SBP, DBP, fasting blood glucose and fasting insulin, whereas no significance was found in the correlation of the increased TG or HDL-C with CIMT thickening.

Hyperlipidemia could lead to increased vascular permeability due to the injury to vascular endothelial cells and focal sloughing and the transfer of plasma lipoprotein into intima could induce ingesting response of macrophages and proliferation of smooth muscle cells in the intima of vessel walls, leading to the formation of plaques. ${ }^{[7]}$ Hyperlipidemia could also lead to the dysfunction of blood coagulation and fibrinolysis, promoting the formation of arterial thrombosis. Moreover, hyperlipidemia could also contribute to arterial wall lipidoses and promote the formation of foam cells. ${ }^{[8]}$

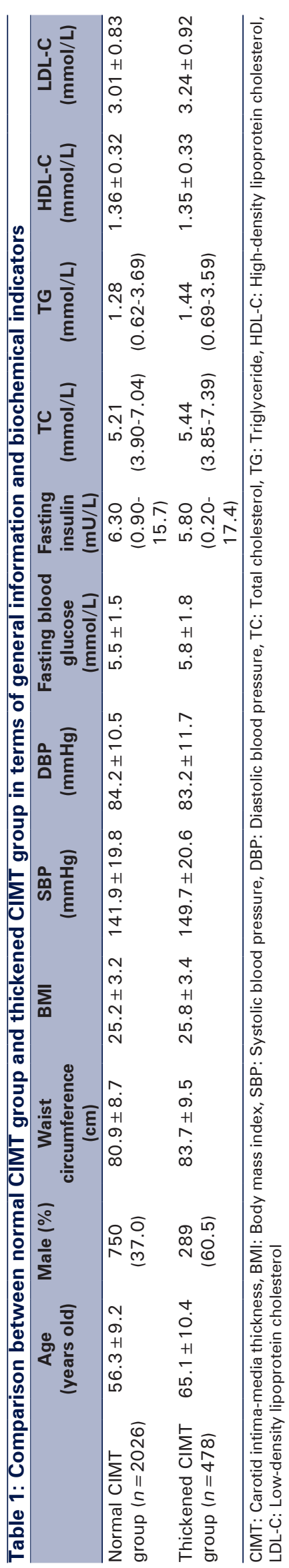




\section{CONCLUSION}

Our study found a significant correlation between dyslipidemia and CIMT thickening in Shanghai urban residents over 40 years old, especially the significant correlation of increased TC and LDL-C with CIMT thickening. For people with dyslipidemia and people with increased TC and LDL-C, regular health check should be performed, especially the carotid ultrasound, in order for early identification, prevention and delayed development of atherosclerosis.

\section{REFERENCES}

1. Alipour A, Elte JW, van Zaanen HC, Rietveld AP, Castro Cabezas M. Novel aspects of postprandial lipemia in relation to atherosclerosis. Atheroscler Suppl 2008;9:39-44.

2. Aslam F, Haque A, Lee LV, Foody J. Hyperlipidemia in older adults. Clin Geriatr Med 2009;25:591-606, vii.
3. Lu EX, Ren WD. Atlas of Vascular Ultrasonic Diagnosis. Shenyang: Liaoning Science and Technology Press; 2001. p. 34-5.

4. Uno K, Nicholls SJ. Biomarkers of inflammation and oxidative stress in atherosclerosis. Biomark Med 2010;4:361-73.

5. Yamasaki Y. Evaluation of carotid artery lesion by echography. Nihon Rinsho 2004;62:87-96.

6. Morrison KM, Dyal L, Conner W, Helden E, Newkirk L, Yusuf S, et al. Cardiovascular risk factors and non-invasive assessment of subclinical atherosclerosis in youth. Atherosclerosis 2010;208:501-5.

7. Gnasso A, Pujia A, Irace C, Mattioli PL. Increased carotid arterial wall thickness in common hyperlipidemia. Coron Artery Dis 1995;6:57-63.

8. Sima AV, Stancu CS, Simionescu M. Vascular endothelium in atherosclerosis. Cell Tissue Res 2009;335:191-203.

How to cite this article: Guan YY, Li M, Chen XO, Yang W, Tang MM, Chen YH, Zhang YF. Association between blood lipid levels and carotid intima-media thickness in Shanghai urban population. J Transl Intern Med 2013; 1(1): 36-9.

Source of Support: Supported by Major Programs of Science and Technology Commission of Shanghai (09DZ1950200). Conflict of Interest: None declared 